\title{
Germanica
}

\section{Bertolt Brecht en quête d'une musique épique}

Bertolt Brecht auf der Suche nach einer epischen Musik

\section{Joseph Kölbl}

\section{CpenEdition}

\section{Journals}

Édition électronique

URL : http://journals.openedition.org/germanica/1515

DOI : 10.4000/germanica. 1515

ISSN : 2107-0784

Éditeur

Université de Lille

\section{Édition imprimée}

Date de publication : 30 juin 2005

Pagination : 81-99

ISBN : 9782913857155

ISSN : 0984-2632

\section{Référence électronique}

Joseph Kölbl, «Bertolt Brecht en quête d'une musique épique », Germanica [En ligne], 36 | 2005, mis en ligne le 10 juillet 2012, consulté le 06 octobre 2020. URL : http://journals.openedition.org/germanica/ 1515 ; DOI : https://doi.org/10.4000/germanica.1515

Ce document a été généré automatiquement le 6 octobre 2020.

(C) Tous droits réservés 


\title{
Bertolt Brecht en quête d'une musique épique
}

\author{
Bertolt Brecht auf der Suche nach einer epischen Musik
}

Joseph Kölbl

À la mémoire de mon cher collègue

Fred Fischbach.

1 Les livres, les articles consacrés aux musiciens qui ont collaboré avec B. Brecht sont légion. En revanche, on s'est moins souvent posé la question inverse, celle de savoir quelles sont les exigences qui découlent de sa conception théâtrale et s'imposent d'emblée à tout compositeur qui cherche à mettre ses textes en musique. Dans ces pages, nous tenterons d'emprunter cette voie et nous interrogerons, dans un premier temps, sur les principales caractéristiques de ce que l'on appelle communément le « théâtre épique ».

2 Cette nouvelle conception, il commence à la développer à partir de 1927, après avoir écrit «Mann ist Mann »; elle atteindra sa forme définitive vers 1933, au moment où il sera contraint de fuir l'Allemagne à la suite de l'installation au pouvoir du régime national-socialiste.

Or, la genèse du théâtre épique va de pair avec la découverte du marxisme. Certes, Brecht avait déjà réclamé, bien avant, un renouveau de l'écriture théâtrale et de la dramaturgie - d'ailleurs, le terme « épique » apparaît dans ses écrits dès 1926. Mais la terminologie reste quelque peu vague et les concepts sont juxtaposés - exprimant plutôt les intuitions de l'auteur - sans lien organique avec une conception d'ensemble. Ce n'est que le contact avec les écrits de Marx qui déclenchera chez lui une véritable prise de conscience. Dans un texte célèbre, il décrit lui-même dans quelles circonstances cette découverte eut lieu: «Pour une certaine pièce de théatre j'avais besoin, en arrière-plan, de la Bourse aux céréales de Chicago. Je pensais me procurer rapidement les connaissances nécessaires en me renseignant auprès de spécialistes et d'usagers. L'affaire prit une autre tournure. Personne, ni quelques économistes connus ni les marchands [...], ne sut m'expliquer de façon satisfaisante ce qui se passait à la Bourse aux céréales. J'eus l'impression que ces affaires étaient tout simplement 
inexplicables, c'est-à-dire qu'elles ne pouvaient être saisies par la raison, c'est-à-dire encore qu'elles étaient tout simplement déraisonnables. La façon dont les céréales étaient distribuées dans le monde était tout simplement incompréhensible. Vu de n'importe quel point de vue, sauf celui d'une poignée de spéculateurs, ce marché des céréales n'était rien d'autre qu'un marécage. Le drame projeté [Joe le Boucher] ne fut point écrit, en revanche je me mis à lire Marx, c'est alors, alors seulement que je lus Marx. Et alors seulement mes propres connaissances pratiques éparses et mes impressions se mirent vraiment à prendre vie ${ }^{1} . »$

Ce n'est qu'à la lumière de la théorie économique de Marx que Brecht parvient à préciser les notions mises en œuvre précédemment et à formuler de façon cohérente sa théorie d'une dramaturgie révolutionnaire.

5 En premier lieu, Brecht réussit à cerner avec plus de précision le rôle de l'institution théâtrale : la survivance de l'ancien théâtre s'explique, selon lui, par les intérêts de la classe qui fournit la masse des spectateurs des grandes scènes berlinoises et allemandes en général. Si dans les pièces des auteurs classiques l'art est encore intimement lié aux aspirations politiques de la bourgeoisie, celle-ci, une fois solidement établie aux leviers de commande de l'État, perd tout intérêt pour le progrès de la société. L'évolution de l'art se déconnecte alors progressivement de la vie matérielle et ne peut survivre qu'en se cantonnant dans sa propre sphère; il devient art pour l'art où la «beauté », se manifestant par un simple jeu des formes, devient le seul critère esthétique admis. Pire encore : le texte de l'écrivain s'efface derrière la représentation et la mise en scène, dont seules les prouesses du jeu des acteurs captivent encore le public traditionnel. À quoi bon, dès lors, écrire de nouvelles pièces de théâtre, puisque le répertoire existant suffit largement à satisfaire les besoins de ce type de spectateurs?

De cette situation découle une vive polémique que Brecht mène dans la seconde moitié des années 20 contre le « culinarisme »- cette tendance qui se contente d'ingurgiter les œuvres, de savourer des plaisirs sans lendemain, sans rapport avec les préoccupations de la vie quotidienne - et contre son représentant, «ce jouisseur qui se jette sur n'importe quel appât esthétique, en voulant seulement vivre une aventure et peindre les sensations qu'elle lui a fait éprouver ${ }^{2}$ ». Brecht considère le public bourgeois comme irrémédiablement perdu pour le théâtre d'un type nouveau dont il rêve. Son public, il le trouve dans le prolétariat, les classes laborieuses qui se préparent à jouer un rôle historique, pour qui le plaisir esthétique fait partie de la vie quotidienne, l'enrichit et nourrit les combats politiques. Mais il a conscience que ce public prolétarien ne se contentera pas de quelques menus aménagements du théâtre déjà existant. Il ne s'agira pas de le rendre plus attrayant - par l'introduction de la technique moderne (« Neuerungen ») - mais de bouleverser de fond en comble l'institution théâtrale ellemême (« Erneuerung »).

7 Ce renversement - quoique Brecht n'établisse jamais, du moins explicitement, le parallèle - n'est pas sans rappeler l'entreprise philosophique du jeune Marx et de son ami Fr. Engels, notamment quand ils s'attachent à inverser ce rapport purement spéculatif qu'entretenait la philosophie classique avec le monde dont il s'agissait de fournir, a posteriori, le reflet fidèle. Et ils en viennent à reformuler complètement la dialectique hégélienne dont Engels dira que Marx l'a totalement renversée «ou, plus exactement : elle se tenait sur la tête, on [i.e. Marx] la remit de nouveau sur ses pieds ${ }^{3} »$.

Un nouveau rapport qui doit s'établir entre la scène et le public, non plus caractérisé par la réception passive d'impressions propres à émouvoir, est l'élément central de ce 
renversement, celui qui entrainera des conséquences pour l'écriture dramatique, la mise en scène et donc pour le jeu des acteurs, les décors etc. Le spectateur devient ainsi un partenaire à part entière, à qui non seulement s'adresse le texte mais qu'il est appelé à suivre de manière critique. Brecht fait appel à la raison et non à un sentiment diffus d'adhésion; il ne cherche pas à subjuguer le spectateur, mais l'incite à juger l'action dramatique et le comportement des personnages. Le public doit avoir conscience d'être au théâtre, d'assister à une re-présentation qu'il ne faut pas confondre avec la vie réelle : et Brecht d'attaquer le naturalisme qui prétendait montrer le monde «tel qu'il est réellement ", sans aucune adjonction. En réalité, celui-ci se contentait de considérer l'état actuel de la société comme naturel, immuable, la dépendance du milieu dans laquelle vivent les hommes comme éternelle, comme une contrainte absolue. Face à cette situation, la seule réaction possible du spectateur était la pitié, la compassion. Rejetant dos à dos le naturalisme et l'expressionnisme, Brecht explique comment par opposition à ces deux courants littéraires est né le « nouveau théâtre »: "Désormais, le théâtre allait désigner par leurs noms "les puissances occultes qui mènent les hommes" et ces noms étaient des noms d'hommes; il allait révéler que ces puissances occultes n'étaient en fait que des puissances clandestines. Il a démontré que le milieu, l'économie, le destin, la guerre, le droit étaient des pratiques qui avaient des hommes pour origine et que des hommes pouvaient changer. Les forces obscures ont disparu de la scène des théâtres comme elles avaient disparu de la science: les hommes y sont apparus intervenants, dans des situations dont on pouvait avoir une vue d'ensemble ${ }^{4}$.»

Brecht hésitera encore pendant longtemps sur la terminologie $e^{5}$ : ce n'est qu'au début de l'exil - privé désormais d'une scène qui lui aurait permis de poursuivre des expériences pratiques - qu'il eut le loisir de réfléchir plus systématiquement sur l'ensemble de sa conception théâtrale. Jugeant le qualificatif « épique » trop étroit et pouvant prêter à malentendu, il proposa le concept de "dramaturgie non-aristotélicienne", parfois accompagné de deux autres épithètes: antimétaphysique et matérialiste. Dans la Poétique du philosophe grec il réfute avant tout le principe de la catharsis en tant qu'acte psychologique qui entraîne fatalement l'identification du spectateur avec le (ou les) personnage(s) du drame.

10 Le spectateur est invité à participer activement à la recherche d'une solution, à se forger une opinion sur le déroulement de l'action et le comportement des personnages, opinion qui engage sa vie en dehors du théâtre. Son jugement qui pèse le pour et le contre, doit être constamment tenu en éveil.

11 Et par conséquent l'écrivain, lorsqu'il conçoit ses pièces, doit adapter sa stratégie à cette attente, en présentant une situation comme historiquement déterminée, en envisageant sa transformation comme possible. En premier lieu il lui incombe de ménager une certaine distance entre le public et l'action dramatique pour éveiller ce plaisir de suivre l'action rationnellement, de la comprendre, au lieu d'être emporté par ses sentiments. L'acteur ne fait que représenter une scène au lieu de s'identifier à un personnage. Les ruptures, les incises détruisent l'illusion et obligent le spectateur à réfléchir à ce qu'il vient de voir sur scène.

C'est une mauvaise habitude de nos scènes bourgeoises que de brouiller les contradictions et de transformer les bonds par lesquels s'accomplissent des évolutions en de petits pas insignifiants 6 .

12 Et partant cette interruption, l'intrusion de la réflexion dans l'action théâtrale exige des acteurs une nouvelle manière de jouer. Brecht, dans un texte de quatre pages écrit 
en février $1929^{7}$, tente d'expliquer ses exigences : les acteurs, en se produisant devant "un public de l'âge scientifique ", devraient montrer leur savoir, les connaissances qu'ils ont acquises « des rapports humains, des attitudes, des forces humaines »; au lieu de recourir à la suggestion et de créer une « ambiance ». La principale préoccupation de l'acteur ne sera pas tant de rendre compréhensible l'homme qu'il incarne, mais l'action qu'il joue.

13 Ce que Brecht expose ici, n'est rien d'autre que ce qu'il appellera plus tard - seulement après 1933, à notre connaissance - l'effet de distanciation (V-Effekt). Au lieu d'accepter un phénomène comme naturel et donc immuable, il voudrait le présenter comme insolite, suscitant notre curiosité. En les isolant de leur contexte, les choses et les actions acquièrent un caractère singulier, étrange qui déclenche chez celui qui les observe une interrogation. "Distancier un processus ou un caractère, c'est d'abord, simplement, enlever à ce processus ou à ce caractère tout ce qu'il a d'évident, de connu, de patent, et faire naître à son endroit étonnement et curiosité 8 .» Et l'auteur aime à comparer la réaction du spectateur avec celle du scientifique qui refuse de contempler passivement le monde qui l'entoure, le ressent dans son étrangeté et cherche par la réflexion, en émettant des hypothèses, à percer ses mystères. De la même manière, le public doit se transformer en chercheur dans le domaine du fonctionnement de la société, ressentir les rapports entre les hommes dans toute leur opacité (Unklarheit) et se montrer avide de mieux les comprendre. Ainsi il parviendra, en ne les acceptant plus comme naturels, à les maîtriser et à imaginer un monde qu'il est possible de transformer".

D'autre part, Brecht se démarque très nettement de la démarche des dadaïstes et des surréalistes qui eux aussi cherchent à montrer le monde dans ce qu'il a d'étrange et de déconcertant, mais qui restent prisonniers de cette vision mystique puisqu'ils ne font pas appel à la ratio ${ }^{10}$. Le théâtre non-aristotélicien, en revanche, analyse les phénomènes et parvient à des solutions "purement terrestres des difficultés » que l'humanité rencontre au cours de son histoire.

Deux procédés permettent en premier lieu d'obtenir l'effet de distanciation: l'historisation (présenter les actions et les personnages - même ceux de notre temps comme historiques et donc temporels et particuliers) et la littérarisation (par le montage ou l'emploi de la citation, par exemple).

16 C'est dans ce contexte, toujours avec l'idée de briser le flot incessant des événements qui se déroulent sur scène, d'interrompre la succession des épisodes de la fable, que Brecht parvient à une nouvelle conception de «l'œuvre d'art totale " (Gesamtkunstwerk); non point dans l'esprit de Wagner, bien sûr, qui songeait à la fusion complète de plusieurs disciplines artistiques. Au contraire, Brecht conçoit cette pluralité comme une juxtaposition d'éléments distincts. Le travail avec Piscator lui avait déjà appris l'intégration des moyens techniques modernes (éclairage, projection de tableaux, de graphiques ou d'un film) dans le spectacle. Par la suite, il prônera la « séparation stricte des éléments »:

D'une certaine manière, en séparant les éléments artistiques, il [i.e. chacun des artistes] maintient dans son association avec les autres arts l'individualité de son art aussi fermement que les autres maintiennent celle de leur art. Ainsi, la combinaison des arts devient vivante; la contradiction des éléments n'est pas étouffée ${ }^{11}$.

17 Ainsi souligne-t-il que les tableaux de C. Neher pour Mahagonny 
prennent position par rapport aux processus qui se déroulent sur le plateau, de sorte que le glouton réel se trouve assis devant le glouton dessiné. La scène jouée répète de la même façon d'elle-même [sic], par le mouvement, ce qui est incorporé dans l'image. Les projections de Neher sont autant un élément autonome de l'opéra que la musique de Weill et le texte. Elles constituent son matériel d'illustration ${ }^{12}$. pour la primauté du texte ou de la musique, qui secouent l'opéra depuis presque deux siècles. Les différentes contributions artistiques, loin de se confondre en un magma indistinct, sont destinées à se commenter et, de la sorte, s'enrichir mutuellement nourrissant la réflexion du spectateur. Après 1948, installé à Berlin-Est, Brecht appellera de ses vœux « un nouveau rapprochement des arts » et défendra l'idée d'un " collectif d'arts autonomes ${ }^{13}$ », tel qu'il l'avait déjà pratiqué au début des années 30 , dans le film « Kuhle Wampe » en particulier. du théâtre (celui qui refuse d'inviter le spectateur à consommer un événement vécu, mais qui cherche à déclencher chez lui une expérience) est le "Gestus »: le principe selon lequel tous les sentiments, toutes les émotions qu'éprouve chacun des personnages du drame, doivent être extériorisés par l'acteur qui l'interprète, et se traduire par une attitude qui résume en quelque sorte les mobiles de l'action du personnage. Le "Gestus ", écrit Brecht pour définir plus précisément le sens du terme :

Par là nous entendons tout un complexe de gestes isolés les plus divers joints à des propos, qui est à la base d'un processus interhumain isolable et qui concerne l'attitude d'ensemble de tous ceux qui prennent part à ce processus [...] ; ou bien un complexe de gestes et de propos qui, lorsqu'il se présente chez un individu isolé, déclenche certains processus [...]; ou encore simplement une attitude fondamentale d'un homme (comme la satisfaction ou l'attente). Un gestus désigne les rapports entre des hommes ${ }^{14}$

Attardons-nous encore un instant sur la dernière phrase de cette citation pour préciser que seul entre en ligne de compte un gestus ayant une portée sociale et non des gestes ne servant qu'à illustrer une action ou à la rendre plus expressive, ce que Brecht qualifie de "stylisation ». Ainsi un travail exécuté sur scène n'est pas ipso facto un gestus, s'il ne révèle pas les relations concrètes entre les hommes, celles qui accompagnent l'action et la représentent comme un acte d'exploitation ou de coopération par exemple. De ce fait, Brecht en arrive à opposer principe gestuel et principe mimique; ce dernier ne visant qu'à donner plus de vraisemblance au jeu scénique, qu'à renforcer l'illusion et par conséquent à faciliter l'identification de l'acteur avec le personnage représenté. Le principe gestuel en revanche caractérise l'action théâtrale comme un événement particulier, comme étant liée à des circonstances précises, à une époque ou un rapport de forces entre classes sociales qu'il s'agit de faire ressortir.

21 Les paragraphes 70 et 71 du «Kleines Organon", écrit en 1948 pour exposer les principaux traits de sa conception non-aristotélicienne du théâtre, nous permettent d'entrevoir le rôle que la musique est appelée à jouer dans le cadre d'une pièce "épique»:

Chaque gestus montré s'accompagne toujours d'un gestus général, qui est de montrer ce qu'on montre. Ce gestus général est souligné par ces adresses musicales au public qu'on trouve dans les chansons [...] La musique doit refuser absolument l'asservissement qu'on lui réserve d'habitude et qui l'abaisse au rang d'une servante stupide. Elle ne devra pas « accompagner », si ce n'est à la façon d'un commentaire. 
Elle ne devra pas se contenter de « s'exprimer » en dégorgeant simplement les états

d'âme où la plonge le spectacle ${ }^{15}$. expliquer l'articulation entre texte littéraire et musique, telle que le théâtre épique la conçoit.

\section{Les contours d'une musique gestuelle}

Si, dans ce qui suit, nous voulons nous interroger sur la fonction que Brecht assigne à la musique dans sa production littéraire, une précision est d'emblée indispensable : dans la pensée de l'auteur - sans que cela soit toujours dit expressément - il s'agit exclusivement, ou presque ${ }^{16}$, de musique vocale ${ }^{17}$ : un chanteur ou un chœur mixte allant jusqu'à quatre voix.

4 La première exigence - qui semble évidente après ce que nous avons dit de la conception brechtienne du théâtre - est bien sûr la compréhensibilité du texte, ce qui l'amène à lutter contre l'hypertrophie de l'orchestre post-romantique (on pense aux grands opéras de R. Strauss, entre autres !) : l'ensemble instrumental ne doit en aucun cas excéder trente $"$ spécialistes $»^{18}$. Dans la pratique, nous le verrons plus tard, les formations sont encore bien plus réduites.

En outre, il demande que les musiciens soient placés sur la scène; ce qui donne à leur intervention l'aspect d'une prestation préparée et étudiée à l'avance; détruisant l'illusion d'une musique qui viendrait subitement rejoindre une parole trop chargée d'émotion. Au contraire : Brecht tient à ce que les morceaux de musique se détachent nettement des parties récitées du spectacle et recommande aux exécutants d'insister sur les préparatifs qui précèdent leurs interventions. À cela s'ajoute généralement un changement d'éclairage - de préférence une lumière crue et uniformément répartie ${ }^{19}$ pour empêcher que la pensée du public ne divague -, la projection d'un titre ou le grimage du (ou des) chanteur(s) sur scène.

Lorsqu'en 1935 "La Mère » fut créée à New York par la Theater Union, Brecht ne parvint pas à faire valoir ses exigences sur plusieurs points essentiels. Dans une longue critique, il s'explique sur les erreurs de la mise en scène dans le domaine de l'éclairage et de l'intégration de la musique dans le spectacle, en particulier les deux chants « Die Partei ist in Gefahr » et «Lob der Wlassowas " ${ }^{20}$. Ces remarques soulignent, on ne peut plus clairement, l'importance que Brecht attache aux conditions dans lesquelles la musique doit intervenir au cours de la pièce.

Tout en affirmant la liberté des disciplines artistiques, les réflexions de Brecht ne se contentent pas de fixer un cadre, mais suggèrent aussi à ses compositeurs des critères proprement musicaux. On sait que Brecht avait été marqué dans sa jeunesse par différents genres de musique ${ }^{21}$ : son père était un «passionné du chant choral» et possédait une large culture dans ce domaine. On connaît par ailleurs l'influence décisive qu'a eu le choral luthérien sur l'évolution du jeune poète. Lycéen, il avait également pris quelques leçons de violon, pour s'en détourner ensuite avec dégoût, et fréquentait les salles de concert de sa ville natale, au point qu'un jour il avait caressé l'espoir de devenir un grand chef wagnérien (dans sa chambre, la partition du Tristan et une baguette étaient placées sur un pupitre - il en connaissait de larges extraits par cœur et les dirigeait volontiers devant ses amis). 
D'autre part, il affectionnait les fêtes populaires et les foires, avec leurs orgues de Barbarie et leurs chanteurs ambulants. Pour sa première pièce, Baal, il avait lui-même composé les chants, de même qu'il avait mis en musique un certain nombre de poèmes qui entreront plus tard dans son premier recueil publié : les Sermons domestiques (« Hauspostille»).

Mais il n'avait bénéficié d'aucune formation musicale solide, si bien que dès le milieu des années 20 il se tourna vers des compositeurs professionnels qui mirent en musique ses poèmes et qui fournirent la musique de scène pour ses pièces à partir de «Mann ist Mann ». Il n'empêche que Brecht n'a jamais consenti à abandonner entièrement ces questions à ses collaborateurs. D'ailleurs, le travail de conception d'une nouvelle œuvre ainsi que les nombreux remaniements des pièces déjà créées avaient toujours un caractère collectif : au cours des longues (et exténuantes) séances de travail, Brecht, en mainte occasion, s'est permis de suggérer au compositeur l'allure générale d'une mélodie, une formule rythmique. Ainsi, pour ne prendre qu'un seul exemple, le plus célèbre sans doute, les premières notes de la mélodie de la complainte qui ouvre l'Opéra de quat'sous, auraient été conçues par l'écrivain.

Ses choix stylistiques sont assez tranchés. Comme pour bon nombre de compositeurs de l'époque, le rejet du wagnérisme est un élément déterminant. Faisant appel aux sentiments, à l'illusion, cette musique entraine le spectateur vers la rêverie par sa force irrésistible de persuasion. Le flot musical incessant des opéras de Wagner s'impose impérieusement, ne laisse aucun répit, aucune place à la réflexion critique. Brecht perçoit avant tout la dimension politique qui sous-tend la conception musicale :

Bismarck avait fondé le Reich, Wagner l'œuvre d'art totale, ces deux forgerons avaient fondu et martelé, et chacun d'eux avait conquis Paris. Wagner avait contraint les malheureux banquiers de Wallstreet à s'occuper de Wotan, de ses affaires confuses et de mauvais augure. Ces milieux comprenaient qu'on les invitât à n'interroger en aucun cas le sauveur sur ses origines ${ }^{22}$.

Et il n'en va pas autrement pour « une grande partie de la musique contemporaine »de toute évidence Brecht vise ici le post-romantisme, les opéras d'un Strauss ou d'un Pfitzner - : essentiellement introspective, cette musique n'exprime que les états d'âme de l'individu isolé ; elle contamine l'auditeur « qui se transforme en cire entre les mains des magiciens ${ }^{23}$ ", et conditionne ses émotions.

Contournant cette phase de déclin de l'art bourgeois qui sombre dans le culinarisme, Brecht trouve chez les compositeurs de la fin du XviII siècle, et singulièrement chez Mozart, une attitude différente, celle de la bourgeoisie conquérante.

De la même manière, Brecht juge sévèrement les expérimentations de l'École de Vienne et de son chef de file A. Schönberg dont il perçoit moins - sans doute à juste titre - la rupture avec la tradition musicale que la continuité, l'enracinement fondamental de l'esthétique schönbergienne dans la tradition post-romantique et expressionniste ${ }^{24}$. Ce rejet du dodécaphonisme est d'autant plus intéressant que Hanns Eisler était lui-même élève de Schönberg, et l'un des plus éminents, qui a connu très jeune une certaine notoriété avec sa première œuvre, une sonate pour piano (Klaviersonate op.1).

34 Au maître, il reproche d'avoir « mathématisé » l'art musical au point que celui-ci n'était plus accessible qu'à une poignée d'initiés, seuls encore capables de comprendre ses œuvres. Enfermé dans un cabinet secret («Geheimlaboratorium»), Schönberg aurait adopté une attitude élitiste par son refus (trop) radical du culinarisme et, le fuyant, se serait retranché dans une tour d'ivoire et coupé ainsi de la société. "Des œuvres du 
maître toute espèce de politique était absente, on y aurait même cherché en vain une allusion aux mérites de la monarchie; dans celle du disciple ne manquait aucune de ses idées politiques ${ }^{25}$.» Cette dernière phrase est particulièrement intéressante, car elle démontre une fois de plus que Brecht opère une distinction très nette entre innovations purement techniques (Neuerungen) et "Erneuerung ", la remise en cause de la fonction de la musique, de son rôle dans la société considérée comme une totalité. Pour Schönberg, ce qui devait être le moyen d'atteindre un but, s'est transformé en une fin en soi ; la question de la réception par l'auditeur ne se pose pas - sinon en termes de connaissances techniques mises en œuvre: il laisse le soin de percevoir les formes, le déroulement de sa musique à quelques rares experts.

Cependant, Brecht avait noté, non sans une certaine satisfaction, voire avec fierté, que grâce à sa formation rigoureuse, Eisler était « en possession d'une technique hautement développée ${ }^{26}$. Dès lors il faut se poser la question de la modernité des conceptions brechtiennes dans le domaine de la musique. On connaît ce propos célèbre où l'écrivain affirme qu'il « ne s'agit pas de renouer avec le bon vieux temps, mais de nouer des liens avec les sales temps modernes. Il ne s'agit pas de démanteler la technique, mais de la perfectionner $\aleph^{27}$.

Mais c'est surtout dans le débat sur le réalisme dans l'art, déclenché par G. Lukacs au milieu des années 30 que Brecht, se sentant visé par la polémique du philosophe marxiste contre le formalisme, se voit contraint de défendre sa propre vision du réalisme. Celle-ci, précise-t-il, n'implique pas nécessairement la "popularité » (Volkstümlichkeit), si l'on entend par là l'utilisation des seuls moyens techniques traditionnels - Lukacs préconise, dans le domaine de la littérature, le recours à la technique romanesque des grands auteurs du XIXe siècle (Balzac et Tolstoi notamment). Après avoir rappelé que le terme de "Volkstümlichkeit » lui-même était on ne peut plus ambigu ${ }^{28}$, Brecht souligne que cette conception elle-même n'est pas anhistorique, que les formes «traditionnelles » se sont développées à un moment donné de l'histoire et dans un contexte précis :

Nous nous souvenons que ce peuple a été très longtemps empêché par des institutions puissantes de se développer pleinement, qu'il a été bâillonné artificiellement et de force par des conventions $[. . .]^{29}$.

Brecht en revanche considère le peuple non seulement comme le produit passif d'une situation d'oppression, mais aussi comme l'ensemble de ceux qui luttent activement contre leur sort dans une société capitaliste. Ainsi, l'essentiel n'est pas d'être populaire, mais de le devenir: "Inutile d'exiger que des œuvres d'art soient immédiatement compréhensible de ceux à qui il est donné de les voir ; ce n'est pas la condition pour avoir une littérature populaire. Le peuple peut s'emparer d'œuvres littéraires de bien des façons $[. ..] »^{30}$; et Brecht condamne résolument une attitude paternaliste d'une popularité dictée d'en haut qui considère les gens d'en bas comme irrémédiablement ignares et imperméables à la culture. Il ne s'agit pas de conforter le peuple dans sa médiocrité, mais de l'en sortir par l'éducation et la mobilisation politique, comme il l'avait d'ailleurs démontré dans « La Mère ».

Quand il en vient à parler de ses compositeurs, il insiste toujours sur le caractère difficile, inhabituel de la musique écrite pour ses pièces. Concernant la "Mère », l'une des réalisations les mieux réussies du tandem Eisler - Brecht, il affirme : « La musique d'Eisler n'est pas du tout ce qu'on appelle une musique simple. En tant que musique, elle est assez compliquée, et je n'en connais pas de plus sérieuse »; et en commentant la 
musique de Paul Dessau pour la "Mère Courage", il s'exprime en des termes similaires ${ }^{31}$.

En quoi consiste donc la modernité de la musique que Brecht réclame pour son théâtre? S'il refuse l'esthétique schönbergienne, il ne s'est jamais prononcé sur la question de la tonalité : seule la "cantabilité » de la mélodie et l'intelligibilité du texte devaient être assurées. Ainsi voit-on Eisler, compositeur attitré de Brecht depuis le début des années 30, adapter son écriture aux circonstances pour lesquelles il écrit: chœurs d'ouvriers, intermèdes pour les pièces théâtrales ou chants en soliste. Quant aux premiers, ils avaient connu, tout au long de la Première République allemande, un succès grandissant - quantitativement aussi bien que qualitativement -, et leur niveau faisait également l'admiration de plus d'un chef des chorales «bourgeoises » : au répertoire du Deutscher Arbeitersängerbund figuraient les grandes œuvres vocales des XVIII ${ }^{\mathrm{e}}$ et XIX ${ }^{\mathrm{e}}$ siècles.

41 Et la formation des acteurs célèbres qui travaillaient avec Brecht - sans être des musiciens professionnels - comprenait aussi le chant. Dans ces deux cas, les harmonies sont néanmoins assez simples, les accords faciles à analyser dans le cadre du système tonal : accords parfaits, sixtes ajoutées, accords de septième ou de septième diminuée. Mais les enchaînements sont parfois si surprenants que l'impression d'une tonalité bien affirmée a du mal à s'imposer : Eisler, en évitant les cadences habituelles, les degrés attendus, fait un nouvel usage de la tonalité où les repères usuels font défaut à l'auditeur. D'où un climat d'incertitude qui tient le public (et les exécutants) en constant éveil. Cette écriture obéit ainsi au précepte que Brecht donnait aux acteurs : "Sa diction doit se débarrasser du ton psalmodiant et des rythmes fixes [sic!] qui bercent le spectateur au point que le sens se perd $»^{32}$.

Dans d'autres partitions, l'émancipation de la dissonance est plus radicale. Dans « Das Lob der dritten Sache ", Eisler utilise le Sprechgesang - le texte, rythmé, est récité sur une seule note - ; la difficulté à chanter des intervalles est ainsi éliminée ; et partant l'écriture du compositeur se fait plus rigoureuse : contrepoint strict à trois voix, en imitation, à la manière des Inventions de Bach, entre une trompette, un trombone et le piano (qui ne joue qu'à une voix, doublée à l'octave par la main droite).

Là où le compositeur écrit pour un chanteur seul (et de toute évidence un professionnel) comme dans les « Cinq Élégies hollywoodiennes »- nous pensons ici plus particulièrement à la Première Élégie -, Eisler renoue avec l'écriture atonale telle qu'il l'avait rencontrée dans sa jeunesse chez son maître. Mais la façon dont il pratique cette écriture est bien différente du style expressionniste de Schönberg: rythmes simples, redoublements de notes à l'octave (voir les basses de la main gauche), mêmes accords par temps, voire pour une mesure complète, accords brisés à la main droite - ce que Schönberg évitait systématiquement.

Mais les véritables innovations concernent bien davantage les formes musicales, l'instrumentation et surtout le rythme.

Quant au premier point, on constate l'abandon de la forme sonate telle que le xIX siècle nous l'a léguée: celle-ci, accordant une importance croissante au développement, jusqu'à aboutir à la forme cyclique, abolit progressivement les incises, les ruptures, les " respirations ». C'est pourquoi les compositeurs qui écrivent pour les pièces de Brecht préfèrent une succession de strophes et de refrains. Parfois on trouve une écriture contrapuntique (sujet, imitation et utilisation de fragments tirés de ces matériaux), 
dans l'esprit de Bach et d'autres compositeurs de la première moitié du XVIII ${ }^{\mathrm{e}}$ siècle. Et le recours à des danses, anciennes ou modernes (p. ex. le foxtrott), n'est pas rare.

L'instrumentation se caractérise par sa grande économie de moyens: les effectifs oscillent en règle générale entre quatre et huit instrumentistes. Signalons notamment l'absence des cordes frottées (liée à l'aversion que le jeune poète a conçue pour le violon) à l'exception d'une contrebasse dont la fonction essentielle est de renforcer les basses du piano, et, par conséquent, la prédominance des instruments à vent, notamment des cuivres (trompette, trombone...), ainsi que des percussions. Au piano est dévolu un rôle harmonique, mais aussi rythmique (l'emploi fréquent d'accords frappés à la main droite, alternant avec des octaves à la main gauche). Parfois un instrument à cordes pincées, tel le banjo, vient s'ajouter à cet ensemble.

Le rythme frappe en premier lieu par son caractère répétitif - reproduisant en musique, si l'on peut dire, la lumière crue que Brecht réclame pour la scène - qui n'est pas sans rappeler les rythmes obstinés des concertos «baroques " (ceux de Vivaldi ou de Bach), ici mise au service d'une intention argumentative et non persuasive. En revanche, les changements de mesure sont fréquents, dans le but d'adapter la musique au texte. Il est indispensable pour comprendre le rapport entre texte et musique de se pencher, ne serait-ce que brièvement, sur un essai que Brecht a publié en 1939 : «Über reimlose Lyrik mit unregelmä:俤igen Rhythmen ».

Dans cet article, il tente de justifier d'avoir écrit des poèmes sans rime et d'avoir abandonné parallèlement les mètres traditionnels au profit de rythmes irréguliers, « changeants, syncopés et gestuels». Dans les deux cas d'ailleurs l'argumentation est similaire : la rime, "onctueuse » (ölig), habitue trop vite l'oreille à un phénomène répétitif, « elle prête aisément au poème le caractère d'une chose repliée sur elle-même et qui frappe l'oreille sans entrer ». De la même façon un mètre régulier a " un effet assoupissant, endormant $»^{33}$. Toujours soucieux d'éviter une réception mécanique de ses textes qui engendre la somnolence et mène à une consommation passive, il veut garder le lecteur éveillé, susciter en lui des interrogations et des réactions critiques. Pour ce faire, il préfère recourir à la langue courante et à une expression sobre.

Mais au-delà de ces innovations "techniques ", l'originalité, sinon la modernité que Brecht revendique, nous semble résider dans le fait que sa dramaturgie nonaristotélicienne exige pour chaque intermède musical que celui-ci contienne et exprime une attitude, un gestus bien identifiable : le doute, le courage, la résignation etc.; en d'autres termes que le compositeur lui donne une signification. Or celle-ci, si l'on tient compte du postulat d'un "collectif d'arts autonomes", ne coïncidera pas forcément avec le sens, l'intention des passages du texte qui l'entourent. Le morceau de musique ne se limitera pas, en règle générale, à être une simple illustration de la scène précédente ou suivante : pure tautologie, vide de sens, parce qu'elle n'apporte rien de nouveau. Brecht songe plutôt à un dialogue fructueux entre différentes disciplines artistiques (texte dramatique, musique, décor, film, peinture etc.) qui s'interpénètrent et ainsi s'enrichissent mutuellement par leur apport respectif.

Deux exemples pour rendre plus concrètes les intentions de la dramaturgie nonaristotélicienne : Hans Mayer, analysant la fonction du Salomon-Song de la $9^{\mathrm{e}}$ scène de "Mère Courage et ses enfants $»^{34}$ montre comment un interlude musical complète ou même contredit le message véhiculé par une scène théâtrale : lorsque, mendiant devant un presbytère, le cuisinier entonne le Salomon-Song qui s'attache à démontrer que le respect de la moralité a entraîné plusieurs figures historiques (Salomon, César, Socrate 
et saint Martin) à leur perte, la Courage médite sur une proposition que le cuisinier vient de lui faire: mener une vie paisible et dans l'aisance, mais à condition d'abandonner sa fille muette, Katrin. Contrairement au message délivré par le poème, la cantinière prendra sa décision en faveur de sa fille et renonce à l'offre du cuisinier. Et le spectateur s'aperçoit qu'elle brave la mise en garde que contient le texte et découvre chez elle les vertus d'abnégation, de sacrifice et d'amour maternel.

51 Le second exemple, le song «Lob der illegalen Arbeit » de la pièce «Die Maßnahme » mêle plus intimement encore l'expression musicale à la parole, formant un véritable contrepoint : Eisler adjoint au mot "Klassenkampf » un rythme caractéristique - deux croches et une noire - anapeste qu'il réutilise systématiquement dans la suite du morceau, alors que le mot lui-même n'est prononcé qu'une seule fois dans le texte. Mais à travers l'accompagnement instrumental l'idée de la lutte des classes est omniprésente tout au long du chœur - elle pénètre le texte et justifie en quelque sorte le travail dans l'illégalité ${ }^{35}$. On comprend qu'ainsi la musique contribue, par ses procédés propres, à enrichir le texte, à apporter des informations supplémentaires : ce qu'il est impossible à la structure linéaire de la logique discursive de "dire ", la musique y parvient par la technique de superposition. Elle ajoute quelque chose par le contrepoint qui accompagne un élément significatif (ici : un mot) du texte et se combine avec lui ${ }^{36}$.

Il reste cependant à se demander si la musique est effectivement en mesure de créer une signification indépendamment du texte qu'elle utilise. Toute tentative dans ce sens ne se heurte-t-elle pas à ses propres limites ? Claude Lévi-Strauss, faisant un parallèle entre le pouvoir des sons et la logique discursive, a formulé ce paradoxe, nous semble$\mathrm{t}$-il, de manière exemplaire :

La musique n'a pas de mots. Entre les notes, qu'on pourrait appeler des sonèmes (puisque, comme les phonèmes, les notes n'ont pas de sens en elles-mêmes; le sens résulte de leur combinaison), et la phrase (de quelque façon qu'on la définisse), il n'y a rien. La musique exclut le dictionnaire ${ }^{37}$.

Certes! encore faut-il nuancer ce jugement. S'il est vrai que le lexique des langues naturelles repose uniquement sur des conventions partagées par l'ensemble des locuteurs, il n'est pas tout à fait exact d'affirmer que de telles conventions seraient totalement absentes dans le domaine des sons : que l'on songe à la citation musicale ${ }^{38}$, à l'utilisation de certaines formes qui sont connotées parce que fréquemment assimilées, dans l'histoire de la musique, à une situation clairement identifiable ${ }^{39}$ ou encore à certains motifs que A. Schweitzer a identifiés dans l'œuvre de Bach et minutieusement analysés ${ }^{40}$ : tous ces éléments, par leur caractère "conventionnel ", finissent par véhiculer une signification que le compositeur peut utiliser à la manière d'un dictionnaire. Concédons cependant que ce phénomène n'affecte qu'une petite partie du langage musical.

Qu'il nous soit permis d'envisager ici une autre hypothèse : l'élément «significatif» de la musique, s'il ne se situe pas au niveau intermédiaire entre le phonème et la phrase, ne pourrait-il pas être localisé dans une dimension qui dépasse le cadre de la phrase ? Si bien que nous obtiendrions, dans l'ordre croissant, la succession suivante : sonème « phrase » (motif, ligne mélodique, enchaînement d'accords ou autre) - signifié.

Du coup le terme brechtien de "gestus" (rappelons qu'il s'agit d'un ensemble de gestes, d'une « attitude » que veut suggérer l'acteur) révélerait toute sa fécondité. Et les réflexions de Brecht - sur le terrain de l'univers sonore - n'auraient été rien d'autre qu'un combat incessant pour soustraire ce mode d'expression artistique à l'intrusion de 
l'irrationnel, aux pulsions morbides (où l'auraient voulu enfermer bon nombre de compositeurs de la seconde moitié du XIX ${ }^{e}$ siècle et leurs épigones jusqu'au delà de la Première Guerre mondiale), et pour soumettre les émotions à la raison critique.

\section{NOTES}

1. Traduction citée d'après F. Fischbach : L'évolution politique de B. Brecht (1913-1933). Lille (PUL) 1976, p. 59.

B. Brecht : Gesammelte Werke = GW. Frankfurt/Main (Suhrkamp Verlag) 1967. XX, 46.

2. Écrits sur le théâtre. Paris (Gallimard, La Pléiade) 2000. p. 117.

GW XV, 146: « ... den auf ästhetische Reize aller Art fliegenden Genu監menschen, der nur etwas erleben will und nur seine Empfindungen bei seinen Erlebnissen schildern will. »

Dans ce contexte, Brecht utilise entre autres la métaphore de "pays de cocagne » (GW XV, 339 et passim) ou de "boîte à plaisir» (XV, 171); mais la plupart du temps celle de "trafic de stupéfiants » (XV, 305 et 482 ; XVII, 1015-16 où il reproduit dans la note une citation de Freud, allant dans le même sens). Citons encore ce passage de la Préface du Kleines Organon: "In der zeitgenössischen Produktion bezeichnete es [i.e. das neue Theater] die Entleerung von allen Wissenswerten als ein Verfallsmerkmal: es beschuldigte diese Verkaufstätten der Abendunterhaltung, sie seien herabgesunken zu einem Zweig des bourgeoisen Rauschgifthandels. » (GW XVI, 661). C'est nous qui soulignons.

3. F. Engels : Ludwig Feuerbach et la fin de la philosophie classique allemande. Paris (Éditions sociales) 1976, p. 60.

Cf. aussi MEW XIII, 473-4 [«Wenn dadurch (i.e. Hegels Denkweise) auch das richtige Verhältnis umgedreht und auf den Kopf gestellt wurde... »]; et MEW XXIII, 27 ["Sie (i.e. die Dialektik) steht bei ihm auf dem Kopf. Man mu眸 sie umstülpen, um den rationellen Kern in der mystischen Hülle zu entdecken. »].

4. Écrits sur le théâtre (Pléiade), p. 251.

GW XV, 232: «Nunmehr war das Theater daran, die "verborgenen Kräfte, die den Menschen lenken", mit Namen zu nennen, und zwar menschlichen Namen, und die Verborgenen als nur Versteckte zu zeigen. Das Milieu, die Wirtschaft, das Schicksal, der Krieg, das Recht wurden als von Menschen ausgeübte Praxis, die von Menschen geändert werden konnte, nachgewiesen. Wie auf dem Felde der Wissenschaft verschwanden auf dem Theater die dunklen Mächte. Die Menschen traten eingreifend, in überblickbaren Situationen, auf. »

5. En 1927, il en appelle à la création d'un « grand théâtre épique et documentaire, conforme à notre époque ", en 1930 il parlera du "nouveau théâtre collectiviste", en 1931 de la «dramaturgie dialectique » et, deux ans plus tard, de "dramaturgie révolutionnaire ». Mais il faudra attendre 1939 pour qu'il établisse une distinction claire entre «nichtaristotelische Dramatik » et "style épique » qui, engageant la mise en scène et le jeu des acteurs, découle logiquement de la première.

6. Écrits sur le théâtre, Paris (L'Arche), 1972, II, 592.

GW XVII, 1293 : «Es ist eine üble Gewohnheit unserer bürgerlichen Bühnen, die Gegensätze zu verwischen und die Sprünge, in denen sich Entwicklungen vollziehen, in unbedeutende Schrittchen zu verwandeln. »

7. « Dialog über Schauspielkunst » : GW XV, 188 sqq. ; Écrits sur le théâtre (Pléiade), p. 788-791. 
8. Écrits sur le théâtre (La Pléiade), p. 326.

GW XV, 301 : «Einen Vorgang oder einen Charakter verfremden hei $\mathbb{E}_{2} \mathrm{t}$ zunächst einfach, dem Vorgang oder dem Charakter das Selbstverständliche, Bekannte, Einleuchtende zu nehmen und über ihn Staunen und Neugierde zu erzeugen.»

9. En plusieurs endroits dans les Écrits sur le théâtre, les allusions à la $11^{\mathrm{e}}$ thèse sur Feuerbach de Marx sont d'ailleurs assez transparentes.

Cf. aussi le texte « Marx, der einzige Zuschauer für meine Stücke », GW XV, 129 [Trad. : Écrits sur le théâtre (La Pléiade), p.136], dont voici un extrait: "Aber dieser Marx war der einzige Zuschauer für meine Stücke, den ich je gesehen hatte. Denn einen Mann mit solchen Interessen mu医 seinigen. Es war Anschauungsmaterial für ihn. »

10. Écrits sur le théâtre (La Pléiade), p. 843.

Voir GW XV, 364: "Der Dadaismus und der Surrealismus benutzten Verfremdungseffekte extremster Art. Ihre Gegenstände kehren aus der Verfremdung nicht wieder zurück. Der klassische Verfremdungseffekt erzeugt erhöhtes Verständnis. » C'est nous qui soulignons.

11. Écrits sur le théâtre (La Pléiade), p. 736.

GW XV, 441 : «In gewisser Weise hält er, in seiner Assoziation mit den anderen Künsten, durch eine Trennung der Elemente die Individualität seiner Kunst ebenso aufrecht, wie dies die andern Künste tun. Das Zusammenspiel der Künste wird so ein lebendiges; der Widerspruch der Elemente ist nicht ausgelöscht. »

12. Écrits sur le théâtre (L'Arche), II, 331.

GW XVII, 1012 : « ... nehmen Stellung zu den Vorgängen auf der Bühne, derart, da㹂 der wirkliche

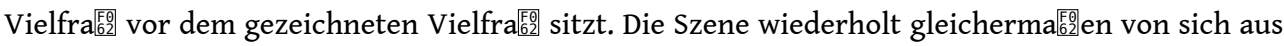
im Flu医, was im Bild steckt. Die Projektionen Nehers sind ebenso ein selbständiger Bestandteil der Oper wie Weills Musik und der Text. Sie bilden Anschauungsmaterial. »

13. Écrits sur le théâtre (L'Arche), II, 518.

GW XVII, 1210 : «Wir erwarten im Sozialismus [...] auch eine neue Annäherung der Künste unter sich. Es handelt sich dabei nicht darum, da熙 das Drama 'sich der Musik' bedienen soll oder die Oper des Textes oder da熙 Drama und Oper durch ein besseres Bühnenbild gewinnen sollen. Sondern in ein und derselben Aufführung soll es drei Behandlungen des Themas geben, durch die Dichtung, durch die Musik, durch das Bild. So entsteht ein Kollektiv selbständiger Künste. » C'est nous qui soulignons.

14. Écrits sur le théâtre (La Pléiade), p. 964.

GW XVI, 753: «Darunter verstehen wir einen ganzen Komplex einzelner Gesten der

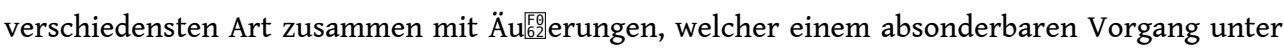
Menschen zugrunde liegt und die Gesamthaltung aller an diesem Vorgang Beteiligten betrifft [...] oder einen Komplex von Gesten und Äu联erungen, welcher, bei einem einzelnen Menschen auftretend, gewisse Vorgänge auslöst [...] oder auch nur eine Grundhaltung eines Menschen (wie Zufriedenheit oder Warten). Ein Gestus zeichnet die Beziehung von Menschen zueinander. »

15. Écrits sur le théâtre (La Pléiade), p. 381.

GW XVI, 697 (§ 71) : «Den allgemeinen Gestus des Zeigens, der immer den besonderen gezeigten begleitet, betonen die musikalischen Adressen an das Publikum in den Liedern [...] Die Musik muß sich [...] durchaus der Gleichschaltung widersetzen, die ihr gemeinhin zugemutet wird und sie zur gedankenlosen Dienerin herabwürdigt. Sie "begleite" nicht, es sei denn mit Komment. Sie begnüge sich nicht damit, sich "auszudrücken", indem sie sich einfach der Stimmung entleert, die sie bei den Vorgängen befällt. »

16. ... à l'exception parfois d'une ouverture, généralement assez courte.

17. Par moments, Brecht semble rejeter toute musique purement instrumentale. Cf. «das konzertante, also nackteste l'art pour l'art » (GW XVII, 1014). 
18. Écrits sur le théâtre (L'Arche), II, 330.

GW XVII, 1011 : « Verkleinerung des Orchesterapparates auf allerhöchstens 30 Spezialisten ». 19. Écrits sur le théâtre (La Pléiade), p. 767.

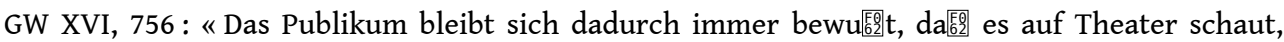
nicht auf wirkliches Leben [...] Das Publikum kommt so nicht so leicht wie bei schummrigem Licht ins Träumen, es bleibt wach, ja wachsam. "

20. Voir Écrits sur le théâtre (L'Arche), II, 374 et aussi 400-1 [GW XVII, 1050 sq. et 1079 sq.].

21. Dümling Albrecht: La Verlag) 1985. pp. 22-72.

22. Écrits sur le théâtre (La Pléiade), p. 727.

GW XV, 486: «Bismarck hatte das Reich, Wagner das Gesamtkunstwerk gegründet, die beiden Schmiede hatten geschmiedet und verschmolzen, und Paris war von beiden erobert worden. Die unglücklichen Wallstreetbankiers waren von Wagner gezwungen worden, sich mit den konfusen und nichts Gutes verkündenden Angelegenheiten Wotans zu befassen. Der Wink, da unter keinen Umständen nach seiner Herkunft befragt werden sollte, fand in diesen Kreisen Verständnis. »

23. GW XVII, 1012 : « Herausstürzend aus dem Untergrundbahnhof, begierig, Wachs zu werden in den Händen der Magier, hasten erwachsene, im Daseinskampf

erprobte und unerbittliche Männer an die Theaterkassen. Mit dem Hut geben sie in der Garderobe ihr gewohntes Benehmen, ihre Haltung “im Leben” ab... » C'est nous qui soulignons.

24. Il mérite d'être souligné que nulle part, tout au long de ses Écrits théoriques, Brecht ne mentionne le nom d'Alban Berg ni, à plus forte raison, son opéra « Wozzeck »! !

25. B. Brecht: Sur le réalisme. Écrits sur la littérature et l'art 2. Paris (L’Arche) 1970, p. 129.

GW XIX, 337 : « Aus den Werken des Lehrers war alles Politische entfernt, selbst Andeutungen, die Vorzüge der Monarchie betreffend, fehlten, in denen des Schülers fehlte kein einziger seiner politischen Gedanken. "

26. Loc. cit., p. 128.

GW XIX, 336 sq. : « im Besitz einer hochentwickelten Technik ».

27. Loc. cit., p. 88.

GW XIX, 298 : «Es wird nicht angeknüpft an das gute Alte, sondern an das schlechte Neue. Es handelt sich nicht um den Abbau der Technik, sondern um ihren Ausbau. »

28. ... usurpé aussi par le national-socialisme (« das Volkstum ») au service de sa mythologie du sang et du sol.

29. B. Brecht: Sur le réalisme. p. 116.

GW XIX, 324 : «Wir wollen uns erinnern, da der vollen Entwicklung zurückgehalten, künstlich und gewalttätig durch Konventionen geknebelt wurde [...]»

30. Loc. cit., p. 124.

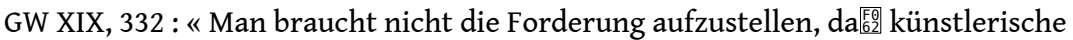

Werke sogleich allen, die sie zu sehen kriegen, verständlich sein sollen, wenn man eine Literatur für das Volk haben will. Das Volk kann sich literarischer Werke auf vielerlei Art bemächtigen...

31. Écrits sur le théâtre (La Pléiade), p. 707.

GW XV, 479: «Die Musik Eislers ist keineswegs das, was man einfach nennt. Sie ist als Musik ziemlich kompliziert, und ich kenne keine ernsthaftere als sie. »

GW XVII, 1135 : «Die Musik Paul Dessaus zur Courage ist nicht hauptsächlich eingängig; wie beim Bühnenbau war auch bei ihr dem Publikum etwas zu tun übriggelassen : das Ohr hatte die Stimmen und die Weise zu vereinigen. Die Kunst ist kein Schlaraffenland. »

Cf. aussi XVII, 1153 sq. (à propos de Lukullus, l'une des productions les plus controversées après le retour de Brecht en RDA) et XVII, 1287 (concernant la musique de Kurt Schwaen pour la mise en scène de « Meister Pfriem »). 
32. Écrits sur le théâtre (La Pléiade), p. 370.

GW XVI, 683 : «Seine Sprechweise sei frei von pfäffischem Singsang und jenen Kadenzen [!!], die die Zuschauer einlullen, so da

33. B. Brecht : Les Arts et la révolution. Écrits sur la littérature et l'art 3. Paris (L'Arche) 1970. p. 30.

GW XIX, 403 : « Der Reim schien mir nicht angebracht, da er dem Gedicht leicht etwas In-sichGeschlossenes, am Ohr Vorübergehendes verleiht. »

«Sehr regelmä̈医ige Rhythmen hatten auf mich eine mir unangenehme einlullende,

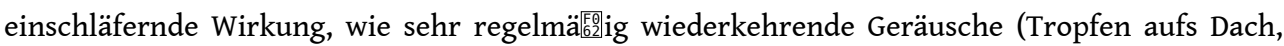
Surren von Motoren), man verfiel in eine Art Trance... »

34. Hans Mayer: Anmerkung zu einer Szene aus «Mutter Courage». In: Anmerkungen zu Brecht. Frankfurt am Main (Suhrkamp Verlag) 1965, pp. 46-55.

35. A. Dümling, loc. cit., p. 301 sq.

36. De la même manière, toute la musique du film «Kuhle Wampe » est un commentaire des éléments visuels - contrastant avec eux pour empêcher l'illusion, et non pas créateur d'illusions. «La musique se fait le porte-parole des protestations contre la situation représentée sur l'écran », commente A. Dümling (loc. cit. p. 319). ["Die Musik artikuliert den Protest gegen die dargestellten Verhältnisse. »]

37. Claude Lévi-Strauss : Regarder - Écouter - Lire. Paris (Plon) 1993, p. 89.

38. Prenons le cas de la Marseillaise qui apparaît en filigrane dans plusieurs œuvres de R. Schumann et qui, dans le contexte de la Restauration, a indubitablement valeur de revendication politique. Que l'on compare ces passages aux références à l'hymne français chez C. Debussy ("Feux d'artifice») ou chez J. Langlais ("Chant héroïque », à la mémoire de Jehan Alain) où cette mélodie à une tout autre signification.

39. Exemple: «La Valse » de M. Ravel qui joue, dans un premier temps, habilement avec les poncifs de l'univers de Strau震 - l'insouciance, la jouissance, la vie sybaritique des Viennois pour le tourner ensuite en dérision par des accords stridents et une forte accélération, montre clairement que cette vie dans l'opulence conduit directement aux horreurs, aux atrocités de la Guerre mondiale.

40. Voir l'analyse du «langage sonore " chez Bach, in Albert Schweitzer : J.S. Bach. Wiesbaden (Breitkopf \& Härtel) 1960 ; et notamment les chapitres XXII («Die musikalische Sprache der Choräle ») et XXIII (« Die musikalische Sprache der Kantaten »).

\section{RÉSUMÉS}

Dans cet article, nous avons voulu nous interroger sur les exigences que Brecht formule à l'égard de tout compositeur qui souhaite "mettre en musique » ses textes dramatiques ou lyriques. Il importait donc, dans un premier temps, de découvrir au sein même de sa conception épique (ou non-aristotélicienne) du théâtre la place qui revenait à la musique. Le concept de « gestus » nous semble central pour expliquer l'articulation qui doit s'opérer entre ces deux disciplines artistiques. Dans la seconde partie de notre contribution, nous avons essayé de montrer comment, à différents niveaux, s'établissent les relations souvent contradictoires entre texte et musique (et d'autres arts) dans le cadre de l'œuvre d'art totale, statut auquel prétendent les drames de Bertolt Brecht. 
In diesem Artikel wurde versucht die Forderungen $\mathrm{zu}$ hinterfragen, die Brecht an jeden Komponisten stellt, der sich an die Vertonung eines seiner dramatischen oder lyrischen Texte heranwagt. Zunächst bestand unsere Aufgabe also darin, den Platz, der der Musik zukommt, im Rahmen der Theorie des epischen (oder nichtaristotelischen) Theaters zu bestimmen. Der Begriff «Gestus » scheint uns zentral, um das Zusammenspiel zwischen diesen beiden Kunstgattungen aufzuzeigen. Im zweiten Teil unseres Beitrages wird versucht darzulegen, wie sich auf verschiedenen Ebenen die oft widersprüchlichen Beziehungen zwischen Text und Musik (und anderen künstlerischen Äußerungen) innerhalb des Gesamtkunstwerkes herstellen, zu dem Brechts Dramen gelangen wollen.

\section{AUTEUR}

\section{JOSEPH KÖLBL}

Université Charles-de-Gaulle - Lille 3 